\title{
Sistema de soporte para la toma de decisiones en el ámbito urbano y regio-nal. Caso de estudio: servicios energéticos de la provincia de Buenos Aires.
}

\section{Decision support system for urban and regional scales. case study: energy services in the province of Buenos Aires.}

\author{
Barbero, Dante Andrés* \\ Instituto de Investigaciones y Políticas del Ambiente Construido. \\ Facultad de Arquitectura y Urbanismo. Universidad Nacional de La Plata. \\ Discoli, Carlos Alberto** \\ Instituto de Investigaciones y Políticas del Ambiente Construido. \\ Facultad de Arquitectura y Urbanismo. Universidad Nacional de La Plata. \\ Martini, Irene $e^{\star \star *}$ \\ Instituto de Investigaciones y Políticas del Ambiente Construido. \\ Facultad de Arquitectura y Urbanismo. Universidad Nacional de La Plata. \\ Ferreyro, Carlos**** \\ Instituto de Investigaciones y Políticas del Ambiente Construido. \\ Facultad de Arquitectura y Urbanismo. Universidad Nacional de La Plata.
}

Fecha de envío: 24/08/2018| Fecha de aceptación: 25/04/2018 | Fecha de publicación: JUNIO 2018

(C) (1) Licencia Creative Commons Esta obra está bajo una Licencia Creative Commons Atribución- NoComercial - Compartirlgual 4.0 Internacional.

\footnotetext{
* Investigador adjunto del CONICET y desarrolla sus actividades en el IIPAC (CONICET / FAU, UNLP). Es egresado de la Universidad Nacional de Córdoba en la que obtuvo el título de Posdoctorado y de la Universidad Nacional de La Plata, en la que obtuvo los títulos de Doctor en Ciencias Informáticas, Licenciado en Informática y Analista de Computación. Ha ganado 2 becas por concurso de la Agencia Nacional de Promoción Científica y Tecnológica (ANPCyT) y participado como autor o co-autor en 3 libros y más de 70 publicaciones científicas.

** Investigador Principal del CONICET. IIPAC/CONICET-FAU-UNLP. Doctor en Ciencias de la Universidad Nacional de Salta. Máster en Ambiente y Patología Ambiental de la Universidad Nacional de La Plata en convenio con la Escuela de los Altos Estudios de Siena, Italia. Ingeniero Mecánico de la UTN, Regional la Plata. Director de proyectos de investigación CONICET, ANPCyT y UNLP; cuenta con 14 libros publicados de diferentes temáticas, y 184 publicaciones científicas de carácter internacional, regional y nacional. Ha asistido como expositor a múltiples congresos desde 1984.

*** Arquitecta, Magister en Políticas Ambientales y Territoriales, en el Instituto de Geografía FILO-UBA. Doctora en Ciencias de la Universidad de Salta. Investigadora Adjunta del CONICET en el IIPAC-CONICET-UNLP. Desarrolla su actividad en investigación en los campos del hábitat, la energía y el ambiente. Cuenta con más de 80 artículos a congresos y en revistas con referato de la especialidad. Ha participado en más de 26 proyectos de investigación CONICET, ANPCyT, UNLP y BID. Es ACD ordinario de la cátedra de Estructuras de la FAU-UNLP. Es Docente - Investigadora Categoría III

**** Arquitecto (UNLP) y Especialista en ambiente y patología ambiental (UNLP / Universidad de Siena, Italia). Ha sido Jefe de Trabajos Prácticos en la cátedra de Instalaciones, en la Facultad de Arquitectura y Urbanismo de la Universidad Nacional de la Plata. Es docente-investigador de la UNLP. Posee amplia experiencia en diseño y modelización energética y ambiental en las diversas escalas del hábitat y en la formulación de instrumentos orientados a la sistematización de información. Cuenta con múltiples publicaciones científicas sobre la temática y con premios nacionales a la investigación.
} 


\section{Resumen}

Este trabajo presenta el diseño, la implementación y el uso de un software que permite construir sistemas de soporte para la toma de decisiones -basados en índices e indicadores cuantitativosen el ámbito urbano regional. Se plantea su aplicación para el estudio de los servicios energéticos en la Provincia de Buenos Aires. El software desarrollado permite mantener la consistencia de los valores de índices e indicadores aún en el caso de modelos sistémicos con ciclos. Además, puede ser acoplado a un SIG para visualizar geográfica y numéricamente los resultados, y mostrarlos en un instante dado (modo ejecutar una vez) u observar dichos valores en función del tiempo (modo monitoreo). De esta manera, es posible observar cómo el cambio en el valor de alguna variable (índice o indicador) afecta al resto de las variables del sistema, no sólo desde el punto de vista numérico sino también geográfico.

Palabras claves: Software de soporte para la toma de decisiones; modelo sistémico; indicadores cuantitativos; SIG; planificación regional.

\section{Abstract}

This paper presents the design, implementation and use of a software that allows to build decision support systems based on quantitative indexes and indicators in the regional urban ambit. Its application is proposed for the study of energy services in the Province of Buenos Aires. The developed software can maintain the consistency in the values between indexes and indicators even in the case of cyclic systemic models. In addition, it can be coupled to a GIS to visualize geographically and numerically the state of indexes, and indicators and display their results in a given time (run once mode) or observe these values versus time (monitoring mode). In this way, it is possible to see how the change in the value of some variable (index or indicator) affects the other variables in the system not only from the numerical point of view but also geographical.

Keywords: Software for decision support; systemic model; quantitative indicators; GIS;regional planning. 


\section{Introducción}

Las políticas neo-liberales aplicadas en la Argentina, cuya expresión máxima se ha alcanzado en la última década del siglo XX, han profundizado la fragmentación económica, social, y territorial; principalmente en las aglomeraciones urbanas en general y en las de nuestra región en particular. Las ciudades forman parte del epicentro de estos cambios, condicionando el estilo de vida; situación que ha acentuado la brecha en términos de calidad de vida urbana entre distintos sectores sociales. En consecuencia es necesario al respecto, producir conceptual y empíricamente instrumentos que faciliten la visualización del estado ambiental y de bienestar en que se encuentran los habitantes de las ciudades. Este es un camino complejo ya que se conjugan diversas dimensiones en el proceso de determinación de mecanismos que lo cualifiquen y cuantifiquen.

El estado actual de la gestión en general muestra una falta de estandarización y sistematización en los diagnósticos, dado que frente a estos desafíos, los planificadores de la región disponen de herramientas heterogéneas que dificultan un análisis científico de la situación de las áreas urbanas y su percepción por parte de la ciudadanía. En consecuencia es claro y prioritario poder contar con mecanismos y técnicas que faciliten la planificación y fundamenten la gestión local, particularmente en aquellas dimensiones que impactan mayoritariamente en la satisfacción de los ciudadanos y puedan ser consideradas en el marco de las políticas de Estado. En este sentido, este software facilitaría el análisis de diferentes escenarios basados en indicadores cuantitativos, manteniendo la consistencia de los valores ante el cambio de uno de ellos; permitiendo, además, obtener una visualización gráfica y territorial.

En este contexto, el trabajo plantea el diseño, la implementación y el uso de un software que permite construir sistemas de soporte para la toma de decisiones -basados en índices e indicadores cuantitativos-. A modo de ejemplo, se aplica al estudio de la cobertura del servicio de gas natural por red en los Municipios de la Provincia de Bs As para los años 2001 y 2010 analizando el porcentaje de cobertura de cada municipio y su cobertura ponderada respecto del total de hogares en la Provincia.

\section{Metodología utilizada en el desarrollo del software}

Para la construcción del sistema de soporte orientado a la toma de decisiones resulta necesario definir los indicadores e índices a utilizar. Un indicador es un parámetro, o un valor derivado de parámetros que señala acerca de, proporciona información sobre, y describe el estado de un fenómeno/ambiente/área, con una significación que se extiende más allá de la que está directamente asociada con el valor de un parámetro (OECD, 1993). Un índice, en cambio, es un conjunto "agregado" o ponderado de parámetros o "indicadores" (OECD, 1993 op. cit). En la planificación urbana y regional, los índices e indicadores son utilizados para describir la situación de diversas dimensiones (por

Figura 1. Actualización automática de indicadores a partir de modificaciones en los datos de base. Fuente: Barbero 2008.
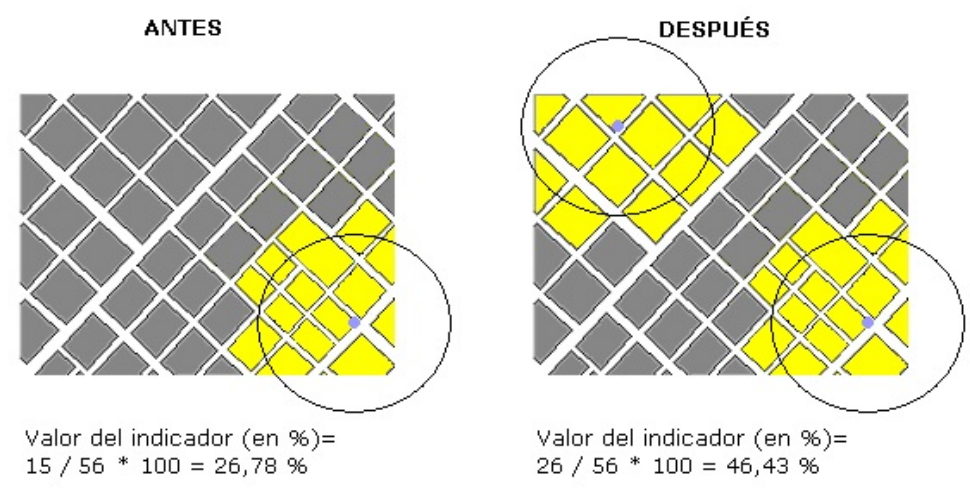
Referencias
Manzanas que están a más de 300 metros de una escuela
Manzanas que están a 300 metros 0 menos de una escuela
- Escuela


Figura 2. Software para el desarrollo de modelos sistémicos basados en índices e indicadores cuantitativos. Fuente: Barbero 2008 op. cit.

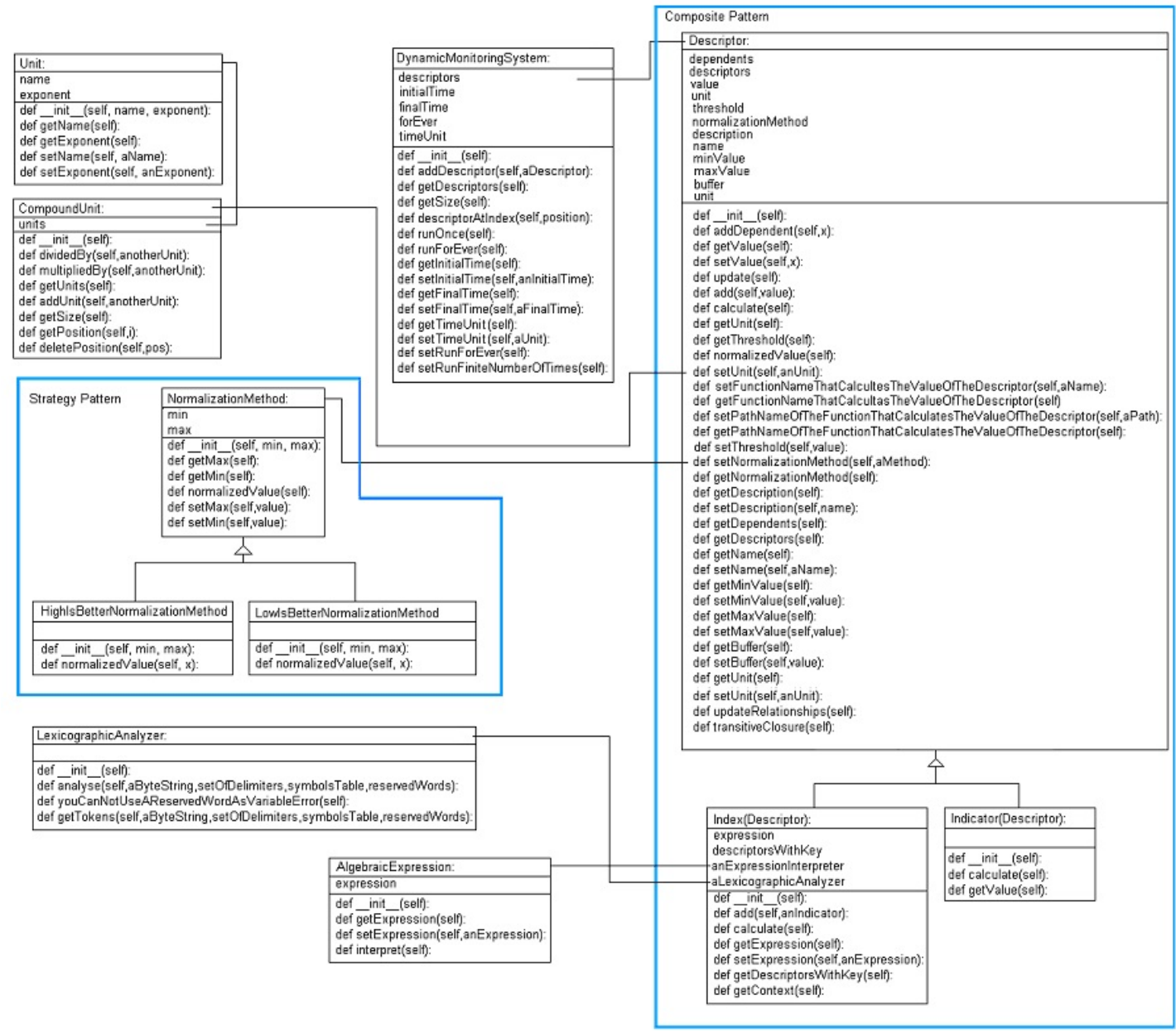

ejemplo: energética, social, de infraestructura, ambiental y económica, entre otras) o variables de interés en modelos que intentan obtener un diagnóstico sobre temas como: calidad de vida urbana (Rosenfeld et. al 2000, Leva 2005), cambio de uso del suelo (Briassoulis, 2000), sostenibilidad urbana (Diputación de Barcelona, 2000), dinámica urbana (Forrester, 1969), entre otros. Ocurre, sin embargo, que en estos modelos la actualización en los datos de base suelen producir cambios en los indicadores, modificando en consecuencia los valores de los índices relacionados. Por lo tanto, es útil poder contar con un software que permita mantener actualizados, y de manera consistente, el valor de los índices e indicadores cuando se produzcan cambios en los datos de base o en el valor de algún índice o indicador del modelo. A modo de ejemplo, y tal como se puede apreciar en la figura 1, un indicador como "porcentaje de manzanas que tienen una escuela a 300 metros o menos" depende de las tablas de manzanas y de escuelas (estas dos tablas son los datos de base a partir de los cuales se calcula el indicador). Así, si se agrega una nueva escuela al tejido urbano, como muestra la figura 1, el valor del indicador cambia (asciende de un $26.78 \%$ a un $46.43 \%$ de manzanas con una escuela a 300 metros o menos). Estas modificaciones suceden a menudo en aplicaciones relacionadas con el catastro y en la planificación urbana y regional por el hecho de ser la ciudad un fenómeno altamente dinámico.

En función de lo expuesto, se planteó el desarrollo de un software que incluye el uso de patrones de diseño (Gamma et al. 1995). Esto se debe a que los patrones de diseño permiten “... desarrollar una forma estandarizada para representar soluciones generales de problemas que se encuentran comúnmente en el desarrollo de software..."(Stelting y Maasen, 2003).

La estructura final del software se muestra en la figura 2, donde se pueden observar los patrones 
Figura 3. Diagrama de clases de las diferentes ventanas de la aplicación y sus interrelaciones. Fuente: Barbero 2008 op. cit.

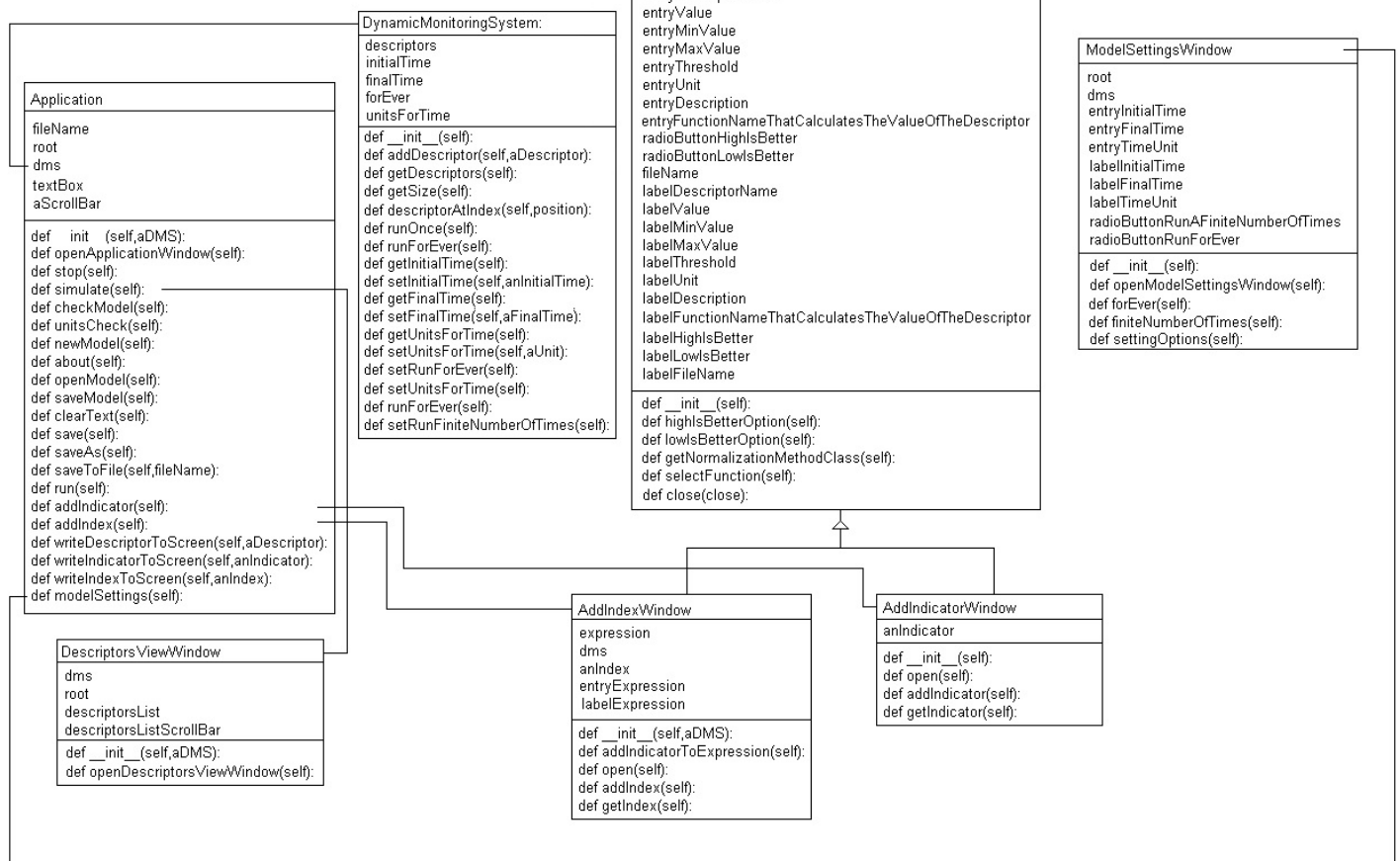

utilizados y las interacciones entre las clases que componen la aplicación. El diseño e implementación de este software ha sido parte de una tesis doctoral (Barbero 2008 op. cit) que trata sobre la representación de modelos sistémicos basados en índices e indicadores cuantitativos usando SIG como herramienta de visualización.

Como se puede observar en la Figura 2, tanto indicadores como índices tienen atributos en común tales como: nombre, valor, valor mínimo, valor máximo, umbral y unidad, entre otros. Los atributos específicos de indicadores e índices se definen en las clases "Index" e "Indicator" respectivamente. La principal ventaja de agrupar las clases de esta manera es la reutilización del código, ya que sólo es necesario declarar los atributos y métodos en común en la clase "Descriptor" en lugar de tener que hacerlo (por duplicado) en las clases "Index" e "Indicator".

Para la modelización de los índices e indicadores se utilizó el patrón "Composite" (Gamma et al., 1995 op. cit) el cual permite la definición de una estructura común para manejar de manera uniforme las operaciones en una jerarquía como la que existe para este caso entre las clases "Descriptor", "Index" e "Indicator". Asimismo, el patrón "Strategy" fue usado para poder establecer el método de normalización que corresponda (recordemos que existen 2 fórmulas posibles: a mayor valor, mejor situación o a mayor valor, peor situación).

Otra parte del software de vital importancia, es el mecanismo de manejo de las dependencias y su actualización; ya que de él depende el correcto funcionamiento del framework. En la solución propuesta, la idea es que cada vez que ocurre un cambio en un índice o indicador, se verifica, qué índices están relacionados de manera directa o indirecta (exceptuando el que sufrió el cambio) y se los actualiza convenientemente.

Dado el carácter genérico del software desarrollado, es posible acoplarlo a un SIG para visualizar geográficamente los cambios que produce la modificación del valor de una variable $y$, al mismo tiempo, observar su efecto sobre el resto de las variables del modelo.

El software tiene una interfase gráfica que facilita la declaración de variables (índices e indicadores) a ser representadas. El diagrama de 
Figura 4. El framework en acción: sus menúes y la generación de código automática. Fuente: Barbero 2008 op. cit.
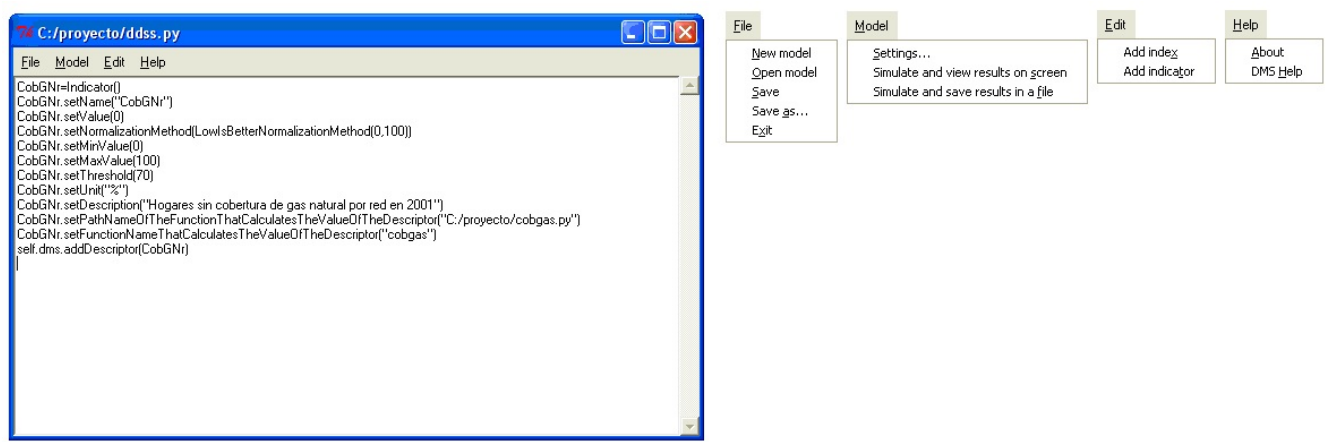

Figura 5. Interfases para el alta de un índice, el alta de un indicador y para especificar los parámetros del modelo. Fuente: Barbero 2008 op. cit.

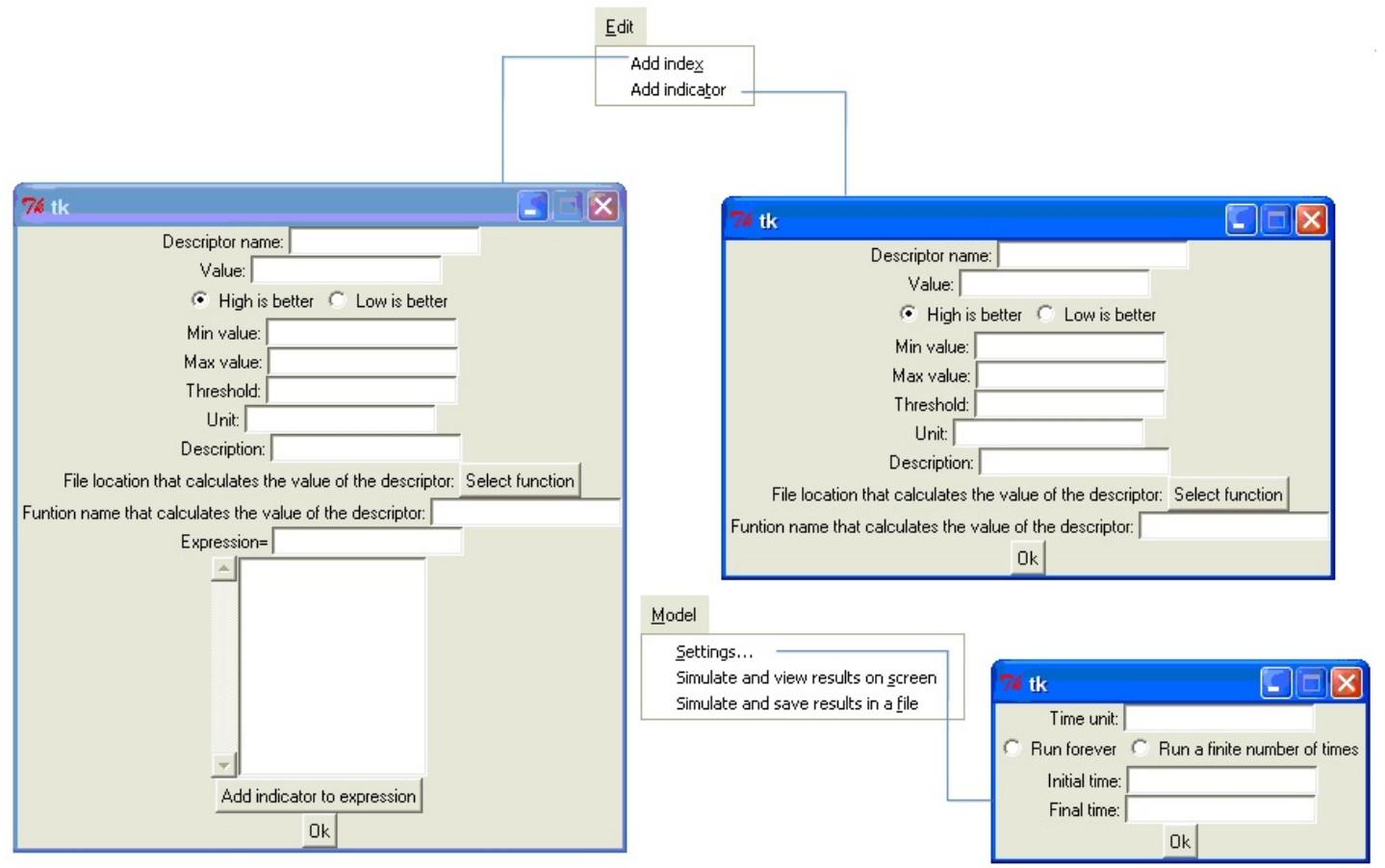

clases de las diferentes interfases y sus interrelaciones se puede observar en la figura 3.

El desarrollo por separado de la interfase con el resto de la aplicación, sumado al uso de técnicas de programación orientada a objetos, permite tener un bajo grado de acoplamiento y un alto grado de cohesión, siendo ambas características muy deseables desde el punto de vista del desarrollo de software. Además, el hecho de haber sido implementado en lenguaje Python, permite trabajar en diferentes sistemas operativos y puede ser acoplado al software ArcGIS $9^{1}$ para vincular los datos geográficos con los valores obtenidos por los indicadores. Dado el carácter genérico del software desarrollado, el mismo puede ser usado en otros dominios diferentes donde la representación de modelos basados en indicadores cuantitativos sea una alternativa válida.

El software se caracteriza por generar código de manera automática. Así, mientras el usuario crea un índice o indicador mediante la interfase

1. ArcGIS 9 es un producto de la empresa ESRI. 
Figura 6. Dos formas de visualizar los resultados numéricos de los indicadores o índices que forman parte de un modelo: por medio de una interfase gráfica o almacenando los valores en un archivo. Fuente: Barbero 2008 op. cit.

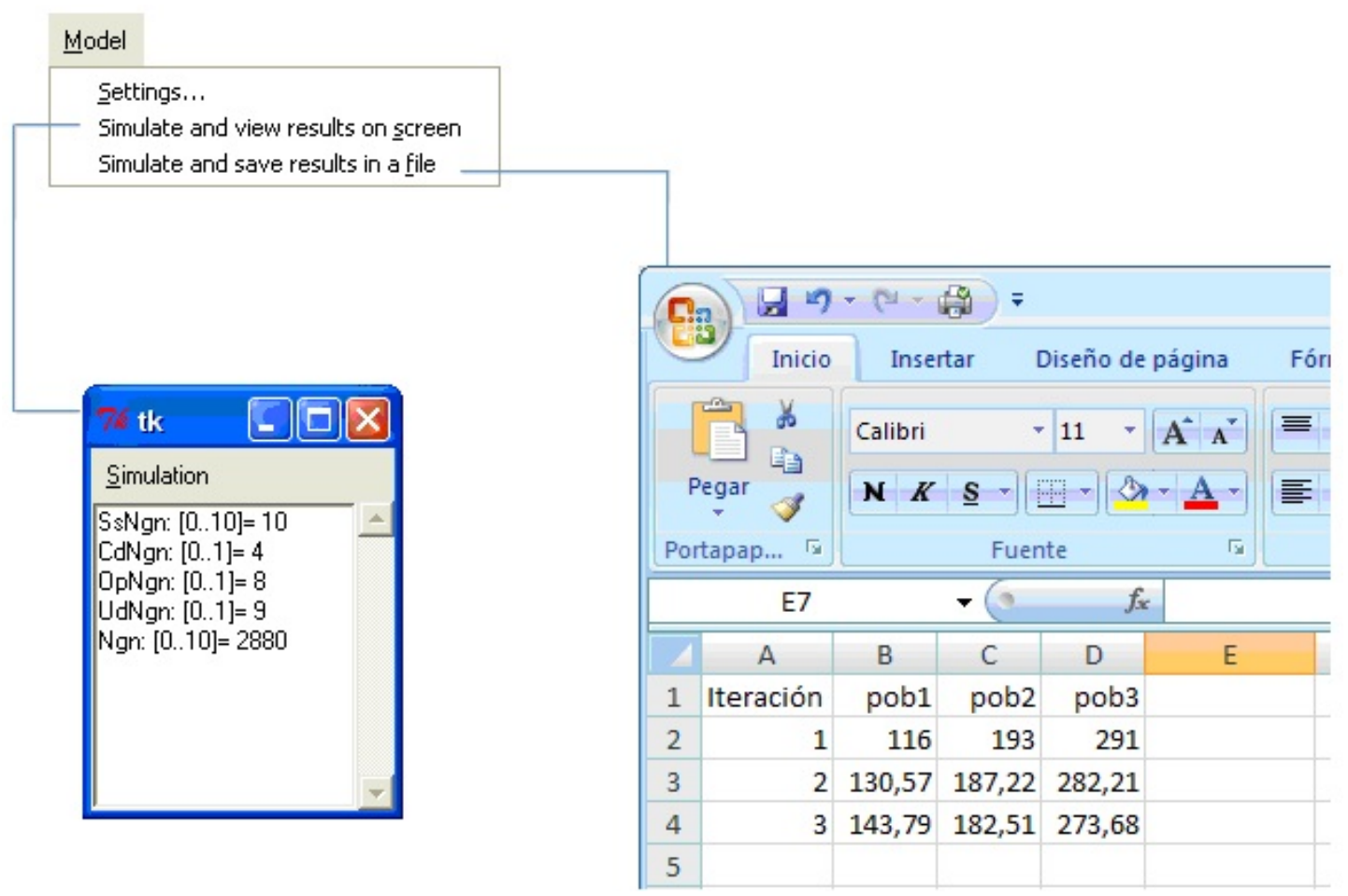

gráfica, el software va generando el código fuente (en lenguaje Python) de modo que puede ser guardado como script evitando de esta manera tener que especificarlo nuevamente cada vez que se quiera utilizar (figura 4).

Se muestra en la figura 4 el código fuente generado automáticamente por el framework para el cálculo del indicador Hogares sin gas natural por red en el año 2001 (CobGNr) en cada municipio.

Para poder usar el framework se deben declarar en primer lugar todos los indicadores que formen parte del modelo a representar, y luego todos los índices. Los índices requieren, además, declarar la expresión matemática que vincula a los diferentes indicadores (eventualmente puede haber índices también) de los cuales depende. Las interfases que permiten la carga de tales datos se pueden observar en la figura 5.

Una vez declarados todos los índices e indicadores del modelo, se debe especificar el modo de ejecución: ejecutar un número finito de veces o ejecutar el modelo en modo de monitoreo. Luego, en función del modo de ejecución, es posible observar, además de los mapas (si los hubiera), los valores de los índices e indicadores en la interfase gráfica o guardarlos en un archivo (figura 6).

\section{Aplicación del software para el análisis del servicio de gas por red en los municipios de la provincia de Buenos Aires}

\subsection{Definición de indicadores para la cons- trucción índices}

A continuación se presentan las fórmulas de los índices que fueron representados en el framework, para analizar la situación del servicio de gas por red en los municipios de la provincia de Buenos Aires (en el período 2001 - 2010).

Para mostrar el Grado de carencia del servicio de gas natural por red de cada municipio (GCSGMj) de la provincia de Buenos Aires se procedió a normalizar los valores en base a la siguiente fórmula:

$$
\operatorname{GCSGM}_{j}=\frac{x-M I N}{M A X-M I N}(1)
$$


donde:

$M A X=$ Número de hogares en el municipio.

MIN = Mínimo número de hogares (0).

$x=$ Hogares sin gas natural por red en el municipio.

$j \varepsilon \quad$ Adolfo Alsina, ..., Zárate\} (Municipios de la provincia de Buenos Aires).

Para calcular el porcentaje de carencia del servicio de gas por red en cada municipio, se normalizaron los valores utilizando la fórmula anterior y se lo multiplicó por 100 para expresarlo en porcentaje. La fórmula resultante es la siguiente:

Porcentaje de hogares sin el servicio de gas natural por red en el municipio j (PHSGMj) $=($ Hogares sin el servicio de gas natural por red en el municipio j) / (Hogares en el municipio j)* 100

$$
\mathrm{PHSGM}_{j}=\frac{\mathrm{HSGM}_{j}}{\mathrm{HSGM}_{i}+\mathrm{HGM}_{i}} * 100
$$

donde:

HSGM $j=$ Hogares sin servicio de gas por red en el municipio j.

HGM $j=$ Hogares con servicio de gas natural por red en el municipio j.

jeAdolfo Alsina, ..., Zárate\} (Municipios de la provincial de Buenos Aires).

Cabe destacar que no es lo mismo que una ciudad pequeña tenga el $10 \%$ de sus hogares sin el servicio de gas, que si lo propio acontece con una ciudad con un gran número de hogares. Por lo tanto, a los porcentajes anteriores hay que multiplicarlos por el número de hogares de cada municipio respecto del total de hogares de la provincia. De esta manera, suponiendo que 2 municipios cuyos nombres sean Municipio $1 \mathrm{y}$ Municipio 2, tienen igual valor en el porcentaje de hogares carentes de un servicio (supongamos por ej.: 20\%), y suponiendo además que el municipio 1 tiene 2.000 hogares, que el municipio 2 tiene 80.000 hogares y que el total de la provincia es de 100.000 hogares; el índice daría como resultado los siguientes valores para cada municipio.

Municipio $1=(2.000 / 100.000)(20)=0,4$. Municipio $2=(80.000 / 100.000)(20)=16$.

Por lo tanto, aún en el caso de que haya 2 municipios con igual valor de déficit del servicio; el municipio con mayor número de hogares carentes del servicio obtendrá un puntaje mayor.
De allí la importancia de este índice, en cuanto a la cobertura de cada servicio, para la toma de decisiones en el ámbito provincial. Por lo antes expuesto, con el objeto de establecer un valor relativo en relación a la cantidad de hogares de cada municipio, es que se plantea el cálculo del porcentaje ponderado de hogares sin servicio de gas por red, a partir de la siguiente fórmula:

Porcentaje ponderado de hogares sin el servicio de gas por red en el municipio j (PPHSGMj) = (Hogares sin el servicio de gas por red en el municipio j ) / (Total de hogares en el municipio j) * 100 * (Total de hogares en el municipio j)/ (Total de hogares de la provincia)

$$
\text { PPHSGM }_{j}=\frac{\text { HSGM }_{j}}{\text { THM }_{j}} * 100 * \frac{\text { THM }_{j}}{T H P}
$$

o lo que es lo mismo:

$$
\text { PPHSGM }_{j}=\frac{\operatorname{HSGM}_{j}}{T H P} * 100
$$

Donde:

HSGM $j=$ Hogares sin el servicio de gas en el municipio j.

THP = Total de hogares de la provincia de Buenos Aires.

THM $j=$ Total de hogares del municipio j. (Es igual a HGSM j + HGM j ).

$T H P=$ Total de hogares de la provincia de Buenos Aires.

$j \varepsilon$ \{Adolfo Alsina, ..., Zárate\} (Todos los municipios de la provincia de Buenos Aires).

A nivel provincial, el porcentaje de hogares que carecen del servicio de gas por red en la provincia (PHSGP) se calcula en base a la siguiente fórmula:

$$
P H S G P=\frac{\left.\sum_{j \in\{\text { AdolfoAl } \sin a, \ldots, \text { Zárate }}\right\}_{j}}{T H P} * 100
$$

donde:

HSGM $j=$ Hogares sin el servicio de gas en el municipio j.

$T H P=$ Total de hogares de la provincia de Buenos Aires.

$j \varepsilon\{$ Adolfo Alsina, ..., Zárate\} (Todos los municipios de la provincia de Buenos Aires).

Las fórmulas 5 y 6 especifican como se calcula el crecimiento del número de hogares entre los años 2001-2010 en la provincia (CNHP) y el 
crecimiento de la cobertura de la red de gas natural en la provincia (CCGP). En ambos casos la unidad es el porcentaje.

$$
C N H P=\left(\left(\frac{T H P 2010}{T H P 2001}\right)-1\right) * 100
$$

Donde:

THP2010 = Total de hogares de la provincia de Buenos Aires en el año 2010.

THP2001 $=$ Total de hogares de la provincia de Buenos Aires en el año 2001.

$$
C C G P=\left(\left(\frac{T H G P 2010}{T H G P 2001}\right)-1\right) * 100
$$

donde:

THGP2010 = Total de hogares con gas por red en la provincia de Buenos Aires en el año 2010.

THGP2001 =Total de hogares con gas por red en la provincia de Buenos Aires en el año 2001.

La fórmula 7 calcula la diferencia entre el crecimiento de la red de gas y el crecimiento del número de hogares. (DCCGPCNH).

$$
D C C G P C N H=C C G P-C N H P
$$

\subsection{Resultados}

A modo de ejemplo, se observan los valores obtenidos por los índices e indicadores, en los años 2001 y 2010, para el partido de Adolfo Alsina (Tabla 1 y Figura 7).
Como se puede observar a partir de los datos de la Tabla 1, para el caso del municipio de Adolfo Alsina, el crecimiento del número de hogares entre los censos 2001 y 2010 fue de un $16,6 \%$. En el mismo período el crecimiento de la cobertura de la red de gas natural fue de 9,68\%. La diferencia entre éste último porcentaje y el correspondiente al crecimiento del número de hogares resulta ser de $-6,92 \%$ lo que significa que el crecimiento de la red de gas estuvo por debajo del crecimiento del número de hogares en el período considerado.

Al especificar el modo de ejecución para que realice los cálculos de los índices e indicadores una única vez, se obtuvieron las salidas (mapas) que se observan en las figuras 7, 8, 9,10 y 11 en tanto que los principales valores numéricos de los índices e indicadores se presentan en la tabla 2. En el caso de que se hubiese elegido el modo de monitoreo, el cambio en alguna de las variables (índices o indicadores) del modelo entre una iteración y otra, hubiese hecho que el framework recalculara (de manera consistente) el valor de aquellas variables relacionadas con la que sufrió la modificación, evitando la tarea de tener que hacerlo manualmente.

Como se puede apreciar a partir de los datos de la Tabla 2, la falta de cobertura del servicio de gas por red en la provincia de Buenos $\mathrm{Ai}$ res fue del $21,6 \%$ en el 2001 y del $35,06 \%$ en 2010.

Tabla 1. Valores de los indicadores en el municipio de Adolfo Alsina.

\begin{tabular}{|l|c|c|c|c|}
\cline { 2 - 5 } \multicolumn{1}{c|}{} & \multicolumn{2}{c|}{ Año 2001 } & \multicolumn{2}{c|}{ Año 2010 } \\
\hline Municipio & Hogares con gas & Total de hogares & Hogares con gas & Total de hogares \\
\hline Adolfo Alsina & 3.862 & 5.349 & 4.236 & 6.237 \\
\hline
\end{tabular}

Tabla 2. Estado de la cobertura de gas por red en los años 2001 y 2010. Fuente: INDEC 2014a e INDEC 2014b.

\begin{tabular}{|l|c|c|c|c|}
\cline { 2 - 5 } \multicolumn{1}{c|}{} & \multicolumn{2}{c|}{ Año 2001 } & \multicolumn{2}{c|}{ Año 2010 } \\
\cline { 2 - 5 } \multicolumn{1}{c|}{} & $\begin{array}{c}\text { Hogares sin gas } \\
\text { por red }\end{array}$ & Hogares & $\begin{array}{c}\text { Hogares sin gas } \\
\text { por red }\end{array}$ & Hogares \\
\hline $\begin{array}{l}\text { Provincia } \\
\text { Buenos Aires }\end{array}$ & 846.079 & 3.917 .739 & 1.679 .040 & 4.789 .484 \\
\hline
\end{tabular}




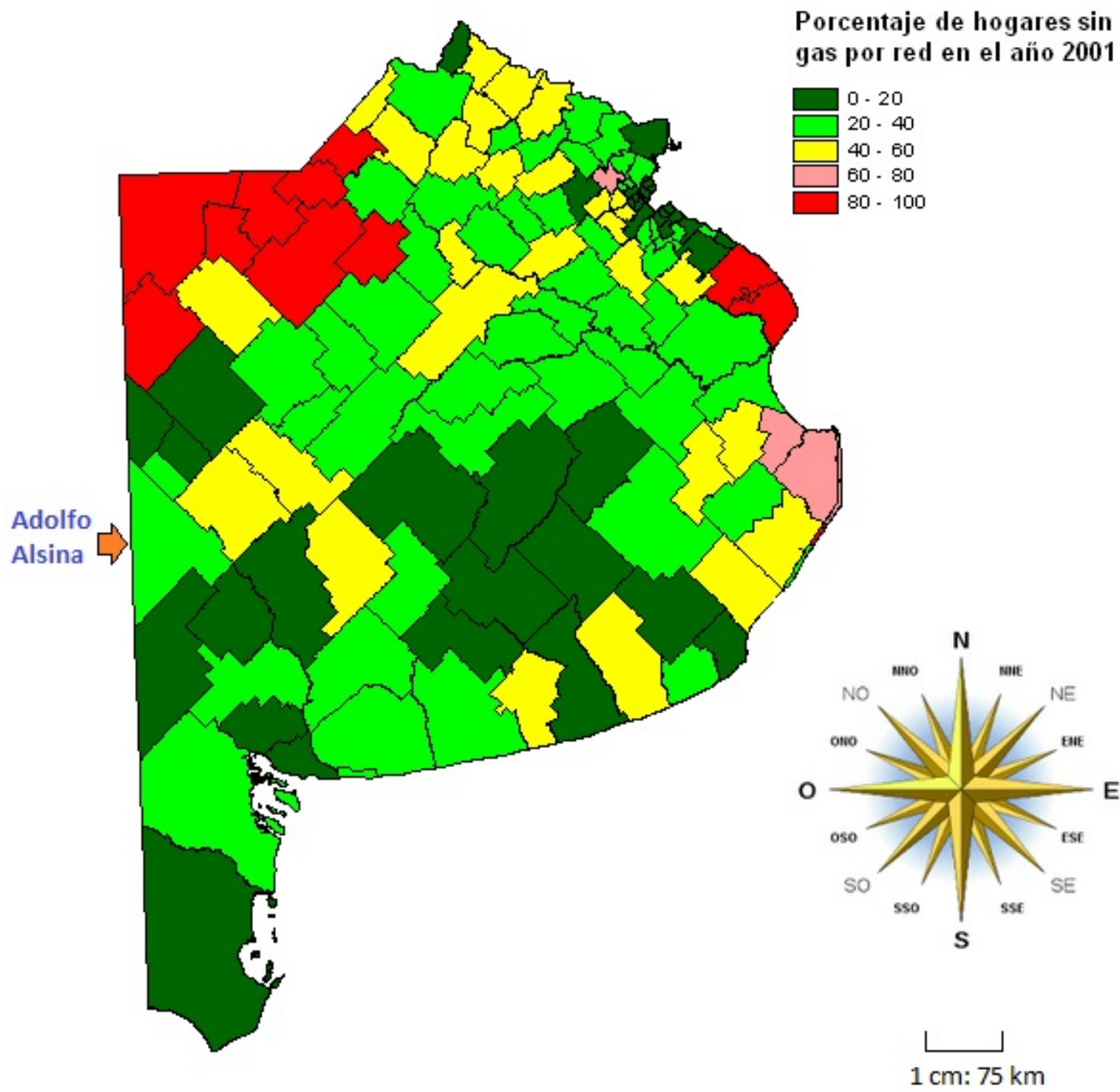

Los mapas de las figuras 7 a 11 sintetizan los resultados obtenidos. Las figuras 7 y 8 muestran el porcentaje de carencia del servicio de gas por red en cada municipio en los censos 2001 y 2010 respectivamente.

Las figuras 9 y 10 muestran los porcentajes anteriores ponderados respecto del total de hogares de la provincia. De aquí resulta que los municipios más afectados (en cuanto al número de hogares) son, en general, los del Gran Buenos Aires.

Por último, la Figura 11 muestra un mapa, mediante una graduación de colores, con aquellos municipios cuyo crecimiento en la red del servicio ha superado el crecimiento de hogares (municipios en color verde) y aquellos municipios en que el crecimiento del servicio ha crecido a un ritmo menor que el número de nuevos hogares.

Así, se puede observar que sólo 12 municipios (de un total de 134) mejoraron la situación en 2010 con respecto al 2001 (diferencia positiva entre crecimiento de la cobertura de gas por red y crecimiento del número de hogares) siendo estos: Alberti, Dolores, General Lavalle, General Pinto, Guaminí, La Costa, Lincoln, Lobería, Carmen de Patagones, Rivadavia, San Cayetano y Tordillo. 
Figura 8. Porcentaje de hogares sin gas por red en el año 2010. Fuente: INDEC. Elaboración propia.

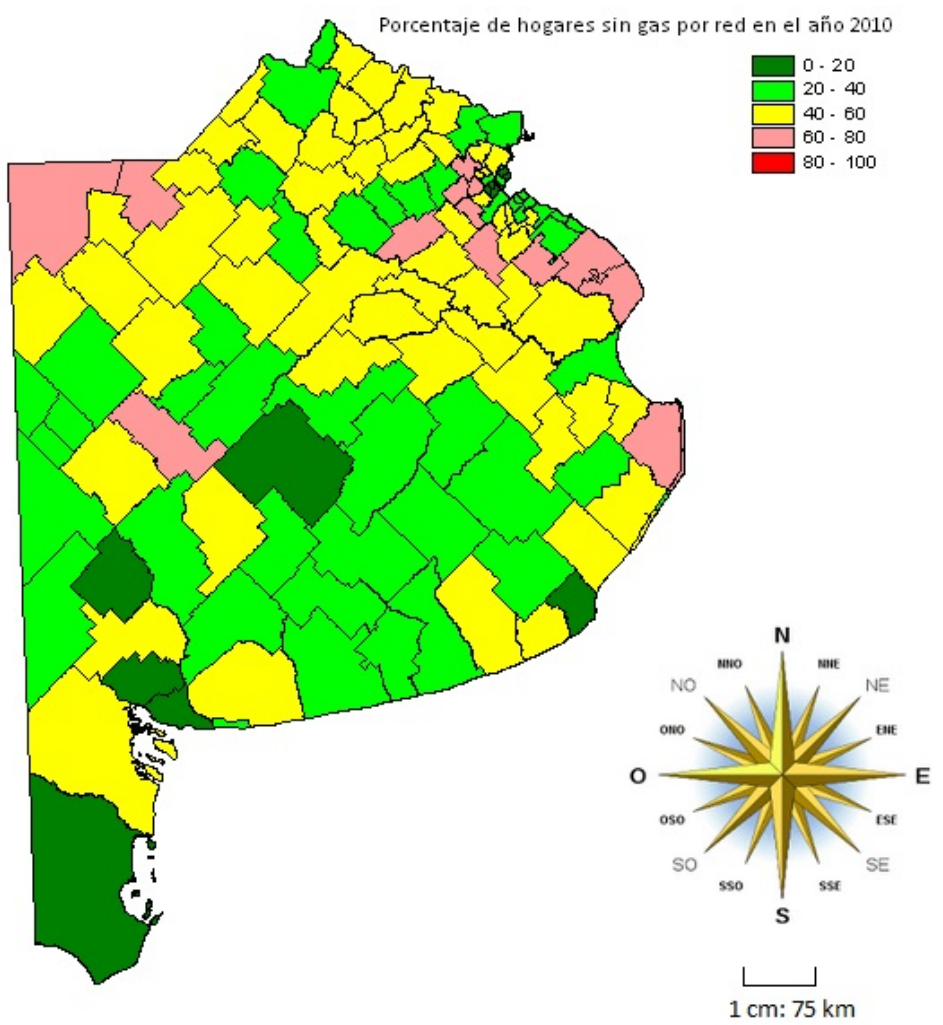

Figura 9. Porcentaje de hogares sin gas por red ponderado por el número de hogares con respecto al total de hogares de la provincia en el año 2001. Fuente: INDEC. Elaboración propia.

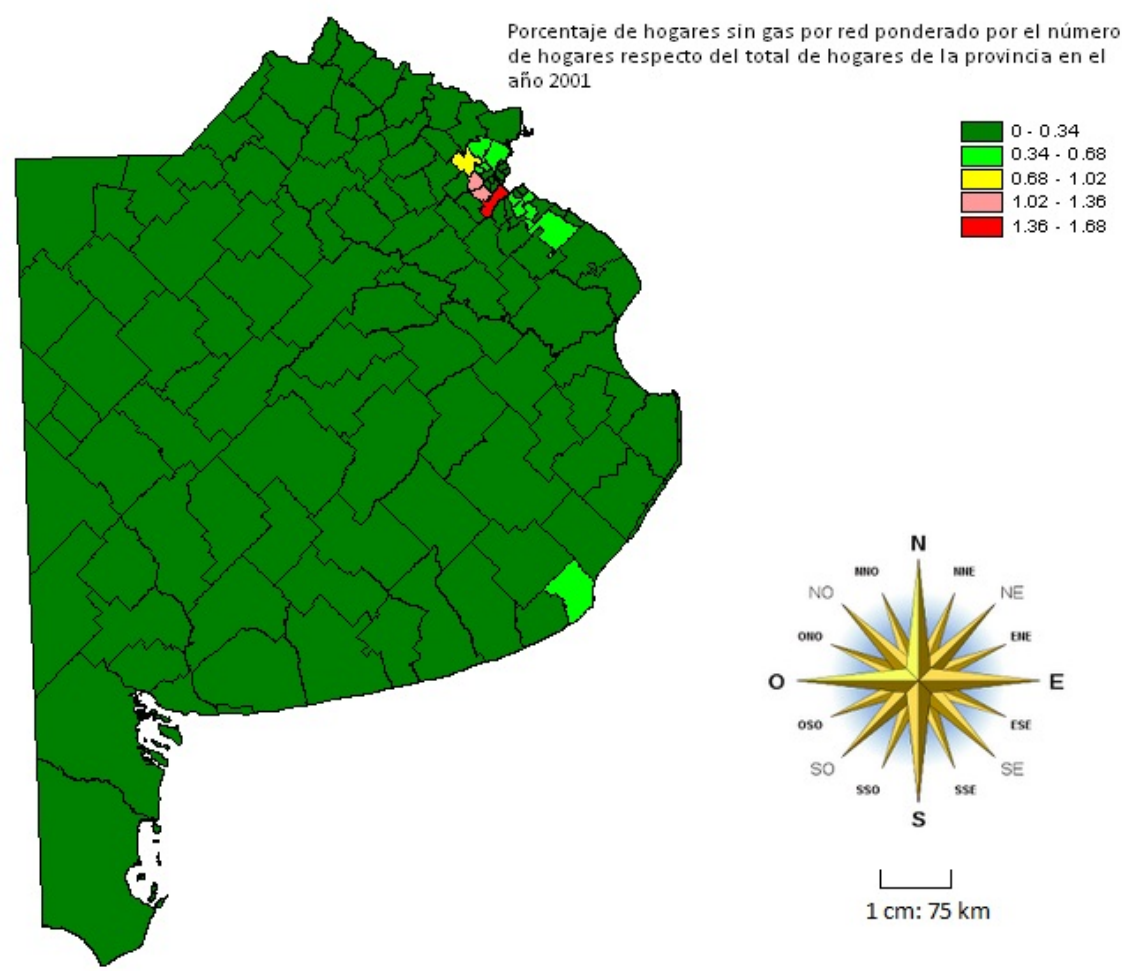


Figura 10. Porcentaje de hogares sin gas por red ponderado por el número de hogares respecto del total de hogares de la provincia en el año 2010. Fuente: INDEC. Elaboración propia.
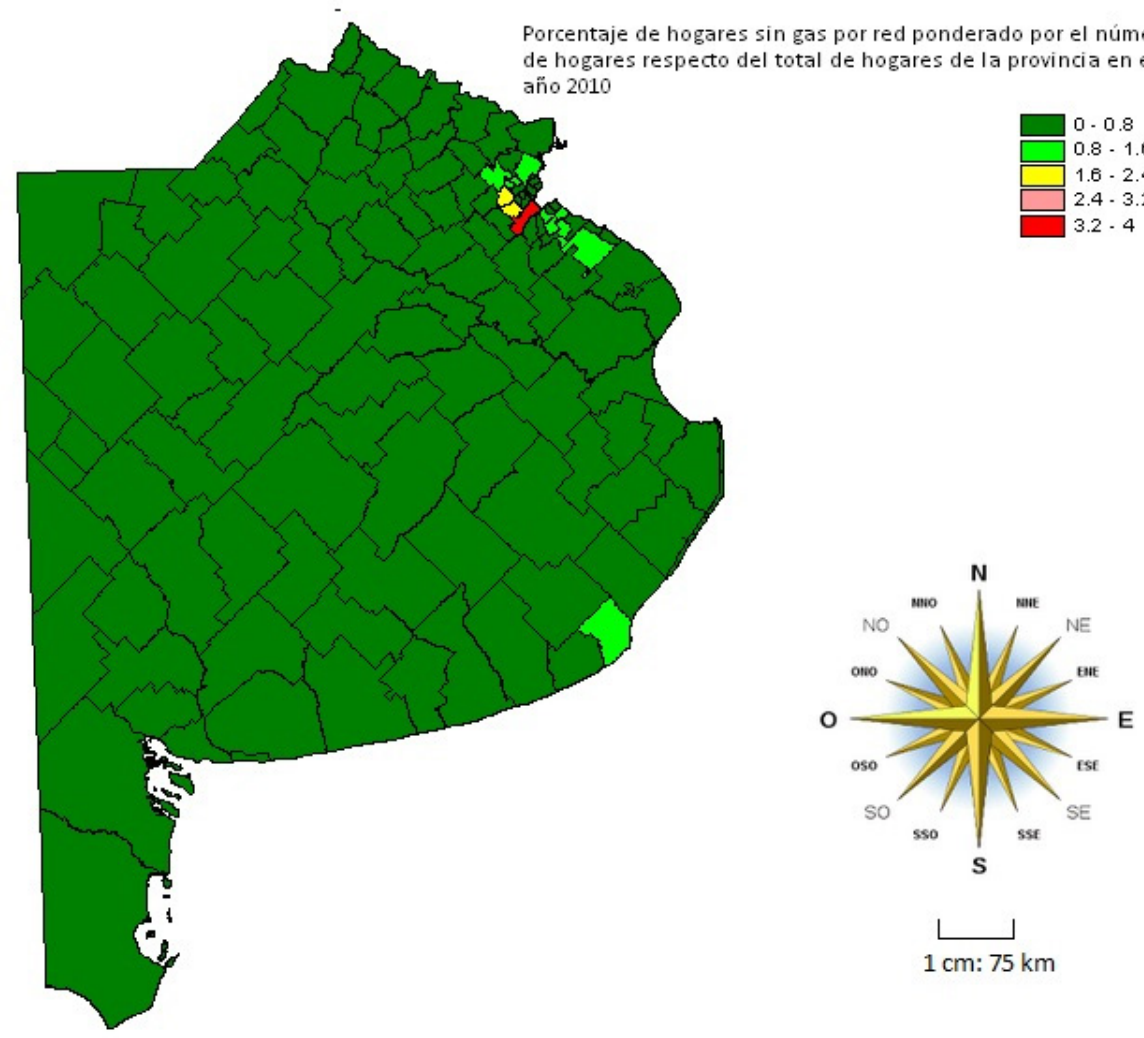

Figura 11. Crecimiento de la cobertura de gas natural por red (\%) - crecimiento del número de hogares (\%) en el período 2001-2010. Fuente: INDEC. Elaboración propia.
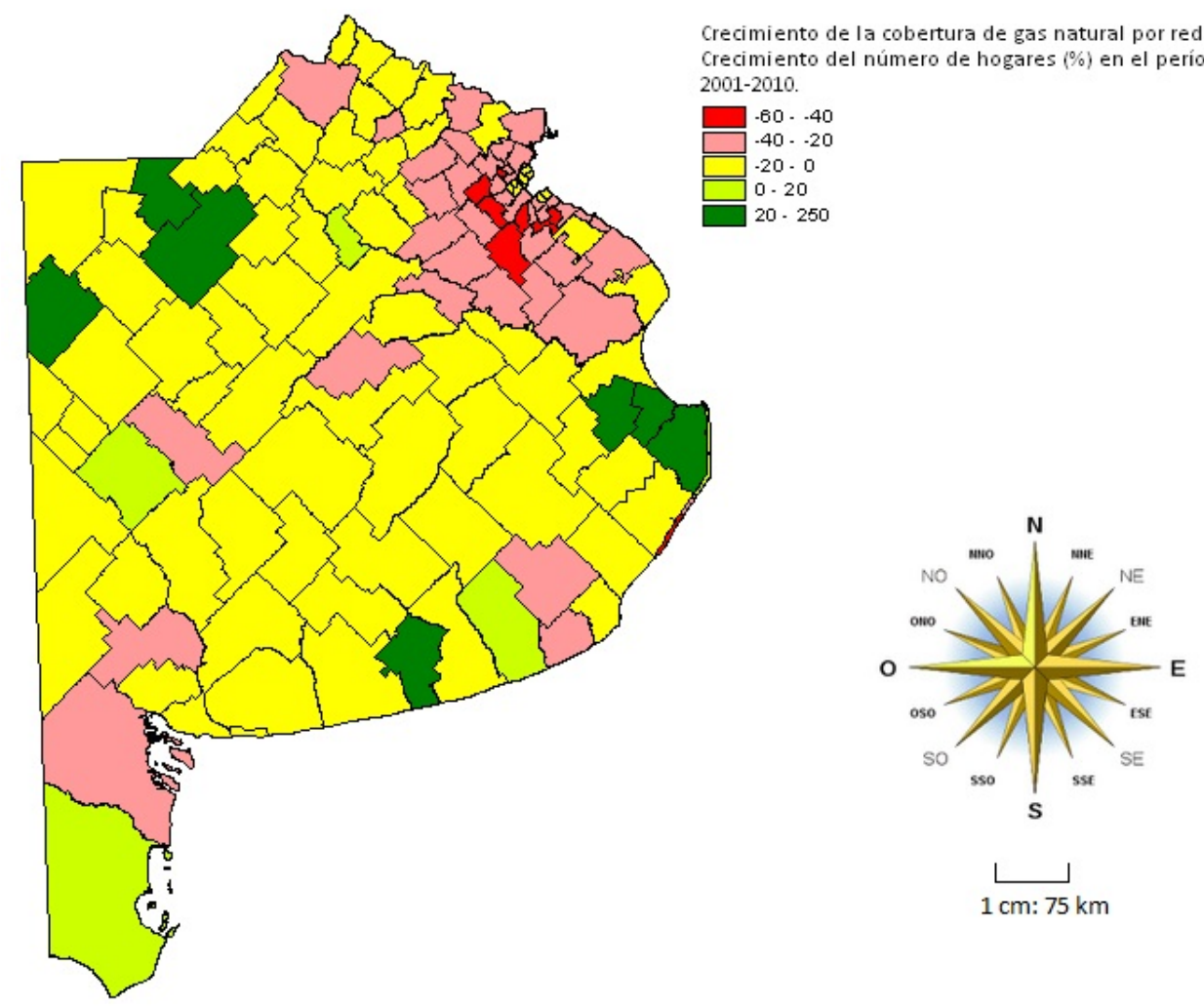


\section{Conclusiones}

Respecto de la utilización del framework para estimar el nivel de carencia del servicio de gas por red en los municipios de la provincia de Buenos Aires, se puede concluir que los resultados obtenidos acerca del grado de carencia en la cobertura del servicio analizado en cada municipio permite alertar de manera temprana a los actores del Estado para iniciar acciones en función del nivel de criticidad observado y de los recursos disponibles. Por lo tanto, el valor de este indicador en un momento dado puede resultar de gran utilidad y servir de apoyo para la toma de decisiones en el ámbito municipal.

Asimismo, los resultados obtenidos con el índice ponderado reflejan la situación de cada municipio con respecto al total de la provincia sirviendo, así, como soporte para la toma de decisiones a nivel provincial.

Con respecto al framework utilizado, éste ha sido aplicado para el análisis de las coberturas del servicio de gas por red y puede ser aplicado también en cualquier otro dominio donde la representación de modelos basados en índices e indicadores cuantitativos sea una alternativa válida. Entre las ventajas de su implementación, se destaca el desarrollo de una interfase gráfica la cual permite ser usada como una aplicación autónoma evitando tener que ingresar instrucciones por teclado. Dicha interfase facilita la declaración de los índices e indicadores que componen el modelo a representar generando un código de manera automática a medida que se van especificando los índices e indicadores del modelo a representar.
Asimismo, permite guardar el código fuente de un modelo en forma de script para su uso posterior, evitando la tarea de declarar el modelo cada vez que sea utilizado. Por otro lado, este software puede ser integrado a un Sistema de Información Geográfica (ArcGIS) permitiendo visualizar geográficamente los cambios que produce la modificación del valor de una variable (índice o indicador) sobre el resto de las variables de un modelo.

En cuanto a los resultados obtenidos en el caso de aplicación analizado, se puede observar que en el censo 2001 la falta de cobertura del servicio de gas por red fue del $21,6 \%$ y en el año 2010 fue de 35,06\%. Es decir, el crecimiento en el número de hogares (se sumaron $22,25 \%$ de nuevos hogares de un censo a otro) creció a un ritmo mayor que la ampliación de la red del servicio (que sumó apenas un $1,26 \%$ de nuevos hogares que cuentan con el servicio de un censo a otro).

Si observamos el porcentaje ponderado, la carencia más importante del servicio de gas por red se da principalmente en municipios del gran Buenos Aires que cuentan con un gran número de habitantes. A nivel provincial el valor obtenido fue de 21,62 \% en 2001 y 35,43\% en 2010. Esto pone en evidencia que cada vez más hogares están careciendo del servicio.

En síntesis, el software adoptado demostró versatilidad en su implementación y puede ser utilizado como herramienta de soporte para la toma de decisiones por parte de los organismos de planificación a nivel municipal y/o provincial.

\section{Bibliografia}

Barbero, D. A. (2008). Modelo sistémico para el manejo con SIG de indicadores de calidad de vida. (Tesis doctoral). Universidad Nacional de La Plata. Facultad de Informática.

Barbero, D. A. Arteaga, A. San Juan, G. A. (2012). Software para el desarrollo de sistemas de soporte para la toma de decisiones en el ámbito urbano. Ponencia presentada en: 7mo Congreso de Medio Ambiente. Asociación de Universidades Grupo Montevideo, La Plata, 22 al 24 de mayo.

Briassoulis, H. (2000). Analysis of land use change. Theoretical and modeling approaches. En: The Web Book of Regional Science. Recuperado de: www.rri.wvu.edu/regscweb.htm. ed., Scott Loveridge. Morgantown, West Virginia University: Regional Research Institute. Consultado: 11-8-2017. 
Diputación de Barcelona. (2000). Sistema municipal de indicadores de sostenibilidad. Barcelona: Diputación de Barcelona.

Forrester, J. W. (1969). Urban dynamics. M.I.T. Press.

Gamma, E. Helm, R. Johnson, R. Vlissides, J. (1995). Design patterns: Elements of reusable object-oriented software. Addison-Wesley.

Instituto Nacional de Estadísticas y Censos. Censo 2001. Recuperado de: http://www.indec.mecon.gov.ar/Webcenso/index.asp. 2014a. Consultado: 29-5-2014.

Instituto Nacional de Estadísticas y Censos. Censo 2010. Recuperado de: http://www.censo2010.indec.gov.ar/ . 2014b. Consultado: 29-5-2014.

Organisation for economic co-operation and development. (1993). Core set of indicators for environmental performance reviews. A synthesis report by the Group on the State of the Environment. Environment monographs No 83.

Rosenfeld, E. San Juan, G. A. Discoli, C. A. (2000). Índice de calidad de vida urbana para una gestión territorial sustentable. Avances en energías renovables y medio ambiente. Volumen 4. Nro. 1. pp. 1.35-1.38.

Stelting, S. Maassen, O. (2003). Patrones de diseño aplicados a Java. Pearson-Prentice Hall. 